\title{
Deep convolutional neural networks for cardiovascular vulnerable plaque detection
}

\author{
Lincan $\mathrm{Li}^{1, *}$, Tong $\mathrm{Jia}^{2}$, Tianqi Meng ${ }^{2}$, and Yizhe $\mathrm{Liu}^{2}$ \\ ${ }^{1}$ College of Mechanical Engineering and Automation, Northeastern University, Shenyang, China \\ ${ }^{2}$ Institute of Machine Learning and Statistics, College of Information Science and Engineering, \\ Northeastern University, Shenyang, China
}

\begin{abstract}
In this paper, an accurate two-stage deep learning method is proposed to detect vulnerable plaques in ultrasonic images of cardiovascular. Firstly, a Fully Convonutional Neural Network (FCN) named U-Net is used to segment the original Intravascular Optical Coherence Tomography (IVOCT) cardiovascular images. We experiment on different threshold values to find the best threshold for removing noise and background in the original images. Secondly, a modified Faster RCNN is adopted to do precise detection. The modified Faster R-CNN utilize six-scale anchors $\left(12^{2}, 16^{2}, 32^{2}, 64^{2}, 128^{2}, 256^{2}\right)$ instead of the conventional one scale or three scale approaches. First, we present three problems in cardiovascular vulnerable plaque diagnosis, then we demonstrate how our method solve these problems. The proposed method in this paper apply deep convolutional neural networks to the whole diagnostic procedure. Test results show the Recall rate, Precision rate, IoU (Intersection-over-Union) rate and Total score are 0.94, 0.885, 0.913 and 0.913 respectively, higher than the $1^{\text {st }}$ team of CCCV2017 Cardiovascular OCT Vulnerable Plaque Detection Challenge. AP of the designed Faster R$\mathrm{CNN}$ is $83.4 \%$, higher than conventional approaches which use one-scale or three-scale anchors. These results demonstrate the superior performance of our proposed method and the power of deep learning approaches in diagnose cardiovascular vulnerable plaques.
\end{abstract}

\section{Introduction}

Vulnerable plaques are unstable plaques which prone to form thrombus. They closely related to the occurrence of acute cardiovascular events such as thrombosis, acute coronary syndrome, coronary heart disease and even sudden death. About 19 million patients die each year due to acute coronary syndrome (ACS) and sudden cardiac death, patients who suffer from myocardial infarction caused by the rupture of unstable coronary atherosclerotic plaques account for $70 \%$ of the total number $[1,2]$. Therefore, accurate detection of cardiovascular vulnerable plaques is significant in preventing cardiovascular diseases.

Developed in recent years, Optical Coherence Tomography (OCT) is a new coronary artery imaging technology both at home and abroad. OCT not only has a resolution rate of $10 \mu \mathrm{m}, 10$ times the resolution rate of IVUS, but also has the ability to identify

\footnotetext{
* Corresponding author: lincanli@outlook.com
} 
organizations (e.g. the main components and characteristics of plaque can be showed in OCT images [3]), hence Intravascular Optical Coherence Tomography (IVOCT) has become preferred method in clinical detection of vulnerable cardiovascular plaques.

In recent years, with the fast development of deep learning and computation power, computer aided diagnosis has been applied to some medical fields, making medical image diagnosis more effective [4]. However, they yield some distinctive drawbacks when it comes to cardiovascular vulnerable plaques detection:

1. Cardiovascular vulnerable plaques adhere to the inner wall of blood vessels, which are more difficult to detect than common object detection targets [5].

2. IVOCT cardiovascular images belong to ultrasonic image. Owing to the imaging process of ultrasonic image, image contents and the interference of instrument catheters, IVOCT cardiovascular images have a lot of speckle noise [6].

3. Medical images require manual annotation by professional physicians, which is more difficult than common object annotation. Besides, the number of available images is limited.

In order to solve these drawbacks mentioned above, we propose a novel cardiovascular vulnerable plaque detection method using deep convolutional neural networks to detect vulnerable plaques. In the proposed method, we first use a Encoder-Decoder structure neural network named U-Net to segment the IVOCT cardiovascular image dataset, removing background and instrument catheters. Then, state-of-the-art object detection framework Faster R-CNN is applied to detect vulnerable plaques. We experiment different threshold values on U-Net and choose the threshold which has best segmentation performance. We also design six-scale anchors for cardiovascular vulnerable plaques detection, so it takes account of a wider range of plaques' size than conventional methods which use one-scale or three-scale anchors.

We evaluate our method on CCCV2017 Cardiovascular OCT Vulnerable Plaque Detection Challenge dataset. The Recall rate, Precision rate and IoU (Intersection-overUnion) rate are $0.94,0.885$ and 0.913 respectively, higher than the $1^{\text {st }}$ team of CCCV2017 cardiovascular OCT vulnerable plaque detection challenge. AP of the designed Faster R$\mathrm{CNN}$ is $83.4 \%$, higher than both conventional approaches and the $1^{\text {st }}$ team of the challenge. Thus the method presented in this paper has great value for clinical application.

\section{Methodology}

In this section, we first give a description of data preprocessing and data augmentation, then describe the two-stage deep learning method presented for cardiovascular vulnerable plaques detection. In stage one, we use U-Net to do segmentation, removing background and instrument catheters in the original images. In stage two, we apply Faster R-CNN to do precise detection. The framework of our proposed two-stage deep learning algorithm is illustrated in figure 1.

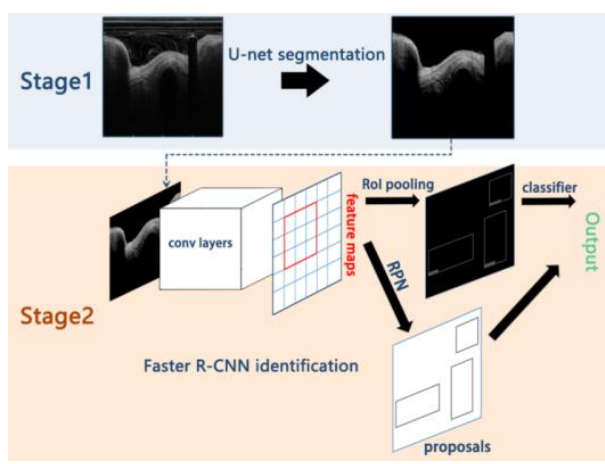

Fig. 1. The framework of our proposed detection algorithm. 


\subsection{Data preprocessing and data augmentation}

The dataset used in this paper is provided by the CCCV2017 cardiovascular OCT vulnerable plaque identification challenge official ${ }^{1}$. The dataset consists of 2000 images, with 1000 positive samples and 1000 negative samples, the size of each image is $720 * 352$ pixels. Ground Truth of the dataset are manually labeled by pathologists. We rotate, translate, shear and zoom the images in a proper range to enlarge the dataset using Keras [7].

\subsection{Formatting cardiovascular image segmentation based on U-Net}

Image segmentation is a crucial step in medical image diagnosis. It can remove unrelated background, instrument catheters and other irrelevant information from the original images, only keeping body organs or tissues which will be detected. Image segmentation is often the first step of computer aided diagnosis algorithm, preparing for subsequent accurate detection [6].

We mentioned in introduction that there are three problems in cardiovascular vulnerable plaque detection, in order to solve these problems, we compare several approaches and adopt U-Net to do segmentation. Proposed by Olaf Ronneberger et al in 2015, U-Net is a Fully Convolutional Neural Network based on encoder-decoder architecture [8]. U-Net won the first place in the ISBI challenge for segmentation of neuronal structures in electron microscopic stacks in 2015 and has been popular since then. Though U-Net has a relatively simple structure, it's able to have an excellent performance even on small datasets [8]. Besides, U-Net has been proved to have better performance on medical image segmentation than any other currently existing convolutional neural networks $[9,10]$.

In this paper, we use U-Net to segment IVOCT cardiovascular images. The insight of U-Net is to classify every pixel in a given image. Suppose the segmentation task require pixels to be classified into $N$ class, when the label of original image is made, each pixel of label should belong to one of the $N$ classes. In the presented method, we classify pixels into two classes. One is human cardiovascular area, the other is unrelated background and instrument catheter area. All labels used in this paper were made by pathologists, they sketched the contours of human cardiovascular area and filled unrelated area into black. Figure 2 gives an example of IVOCT image and it's label. Set $p$ to represent an arbitrary pixel in an image, we normalize $p$ to get $p^{\prime}$ using the equation: $p=p^{\prime} / 255$. Set threshold range as : $0<$ threshold $<1$. If $p^{\prime}>$ threshold, we set the label as 1 , otherwise the label is 0 . Notice that threshold value is directly related to the quality of segmentation results, we make several experiments adjusting threshold values to find the best value. The curve of threshold and validation loss is shown in figure 3. From figure 3. we can observe when threshold $=0.2$ the presented method attain the minimum loss on validation set. We further illustrate the results of U-Net segmentation using different threshold in figure 4.
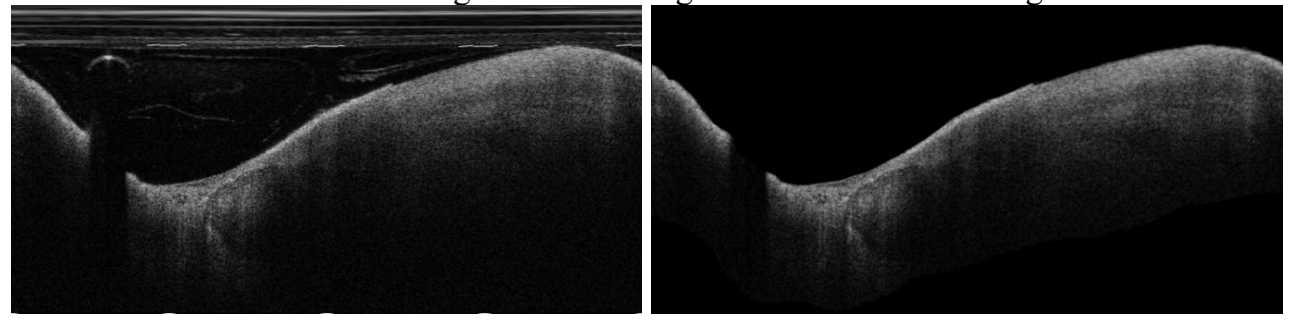

Fig. 2. Left: An IVOCT image from the dataset. Right: Correspondent label of the left image.

\footnotetext{
${ }^{1} \mathrm{http} / /$ ccf-cccv.org/2017/competition3/
} 


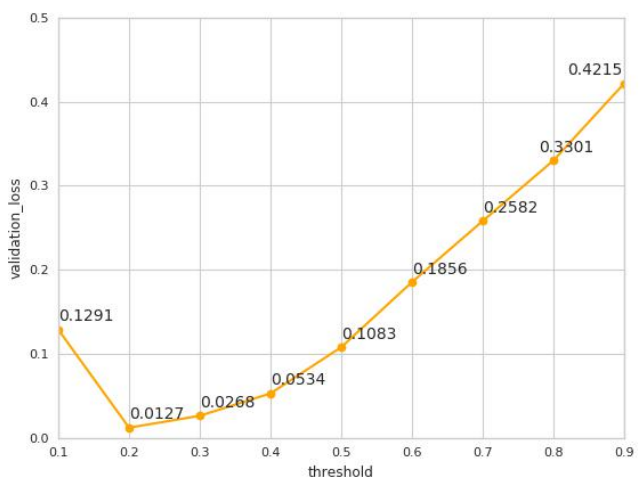

Fig. 3. Curve of threshold and validation loss. We experiment on different threshold values and found validation loss is minimum when threshold $=0.2$.
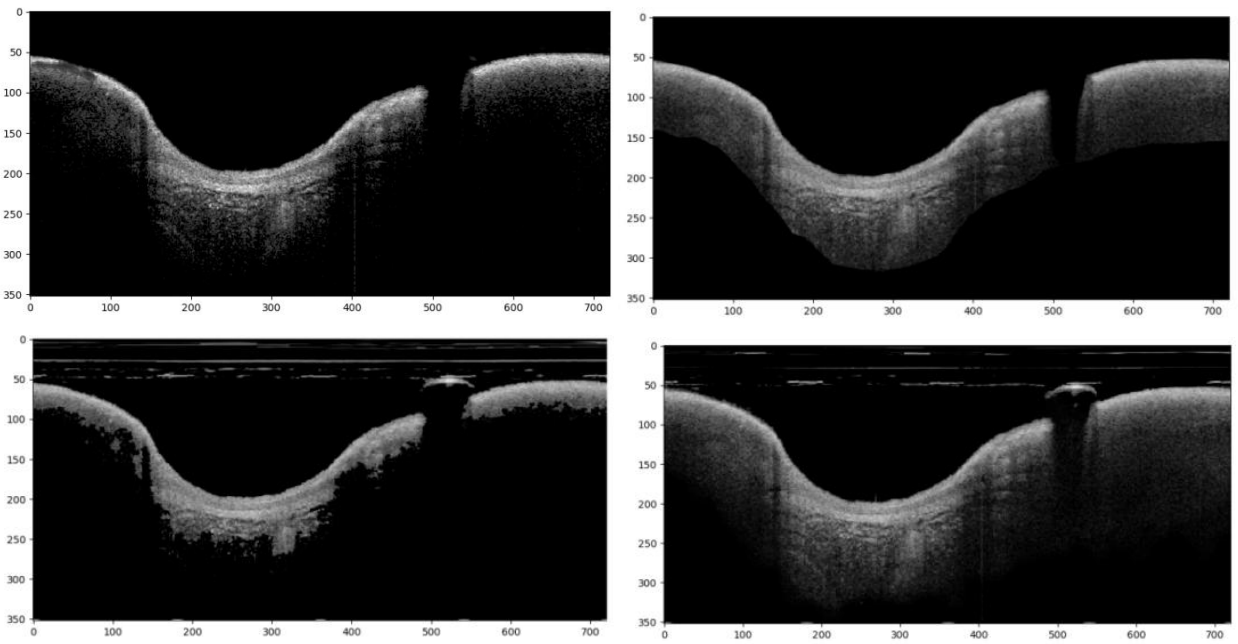

Fig. 4. Comparison of different threshold results. Upper Left: U-Net segmentation result when threshold $=0.2$. It's the best result and we utilize it in our proposed method. Upper Right: Label image manually labeled by pathologists. Lower Left: U-Net segmentation result when threshold = 0.1. Lower Right: U-Net segmentation result when threshold $=0.6$.

\subsection{Precise cardiovascular plaque detection using faster R-CNN}

In this section, we present how we utilize state-of-the-art detection algorithm Faster RCNN to detect vulnerable plaques in IVOCT cardiovascular images which have been operated by U-Net. In detection algorithm, vulnerable plaque regions are marked with rectangle boxes, coordinates of the four corners can be obtained from the label. Since vulnerable plaques vary greatly in size, we utilize six-scale anchors $\left(12^{2}, 16^{2}, 32^{2}, 64^{2}, 128^{2}, 256^{2}\right)$ and compare our six-scale approach with the conventional onescale and three-scale approaches.

Faster R-CNN algorithm consists of two modules:

1. Region Proposal Network.

2. Fast R-CNN [11] detection network.

First, we utilize RPN (Region Proposal Network) to extract detection regions. RPN (Region Proposal Network) can share full-image convolutional features with the detection network [12], making region proposal almost spending no time [12]. The RPN network first extracts features, then generates candidate regions. In the presented method of this paper, 
we utilize six scale $\left(12^{2}, 16^{2}, 32^{2}, 64^{2}, 128^{2}, 256^{2}\right)$ anchors and three aspect $(1: 2,1: 1,2: 1)$ ratios, then classify boxes and refine boxes location.

Second, in order to train the RPN network, we set class labels for each candidate area \{object, non object\}. The label settings for RPN network are described as follows:

Object: candidate regions which have overlap ratio larger than $x$ with arbitrary Ground Truth bounding boxes $(\operatorname{Io} U>x)$.

Non Object: candidate regions which have overlap ratio equal or smaller than $(1-x)$ with all Ground Truth bounding boxes.

Since RPN network extracts a large number of proposed boxes and there are lots of overlapping regions between these proposed boxes, we use NMS (Non-Maximum Suppression, NMS) to merge and delete proposed boxes. To be specific, all candidate boxes are sorted by score and box with the highest score is selected, then we delete boxes which have overlap ratios larger than $x(\operatorname{Io} U>x)$. We then sort proposed regions and take top $N$ proposed regions for detection.

Third, After RPN network got the proposed regions, Fast R-CNN is used to realize the final detection and identification. We train RPN network and Fast R-CNN to let them share the convolutional layers of ResNet101 [13].

\section{Experimental results}

The goal of CCCV2017 Cardiovascular Vulnerable Plaque Detection Challenge is to detect cardiovascular vulnerable plaques in IVOCT images precisely and instantly. Performance of detection algorithms are evaluated by 3 indicators, they are Precision rate, Recall rate and IoU (Intersection-over-Union) rate. We evaluate our presented two-stage deep learning method on the official dataset of CCCV2017 Cardiovascular Vulnerable Plaque Detection Challenge. Let $T P$ be the positive class which the algorithm also predicts as positive class, $F P$ be the negative class which the algorithm predicts as positive class, $F N$ be the positive class which the algorithm predicts as negative class, $S_{g}$ demotes the area of Ground Truth bounding box, $S_{p}$ denotes the area of predicted bounding box. The computational formula of the three indicators are shown below:

Precision rate: $P=T P /(T P+F P)$

Recall rate: $R=T P /(T P+F N)$

IoU rate: $I o U=\left(S_{g} \cap S_{p}\right) /\left(S_{g} \cup S_{p}\right)$

Total score: $\mathrm{S}=0.5 *(2 * \mathrm{P} * \mathrm{R} /(\mathrm{P}+\mathrm{R}))+0.5 * \mathrm{IoU}$

We compare our detection algorithm with other submitted algorithms, the result is illustrated in table 1 and table 2. Table 1 shows the performance comparison of top 3 team and us, we can see our method has the highest Precision rate and IoU rate, thus we get the highest total score. Table 2 compares AP value of our method and top3 team.

Table 1. Performance comparison with the top 3 teams of CCCV2017cardiovascular vulnerable plaque detection challenge.

\begin{tabular}{|l|c|c|c|c|}
\hline \multicolumn{1}{|c|}{ Team } & Precision rate & Recall rate & IoU rate & Total score \\
\hline $1^{\text {st }}$ team & 0.825 & 0.932 & 0.883 & 0.879 \\
\hline $2^{\text {nd }}$ team & 0.865 & 0.838 & 0.869 & 0.860 \\
\hline $3^{\text {rd }}$ team & 0.868 & 0.713 & 0.831 & 0.807 \\
\hline Our & 0.943 & 0.885 & 0.913 & 0.913 \\
\hline
\end{tabular}


Table 2. AP comparison with top3 teams of CCCV2017 cardiovascular vulnerable plaque detection challenge.

\begin{tabular}{|c|c|}
\hline Team & AP(\%) \\
\hline $1^{\text {st }}$ team & 76.3 \\
\hline $2^{\text {nd }}$ team & 72.5 \\
\hline $3^{\text {rd }}$ team & 61.9 \\
\hline Our & 83.4 \\
\hline
\end{tabular}

To better demonstrate the performance of our presented method, we draw ROC curve of our method as it shown in figure 5 .

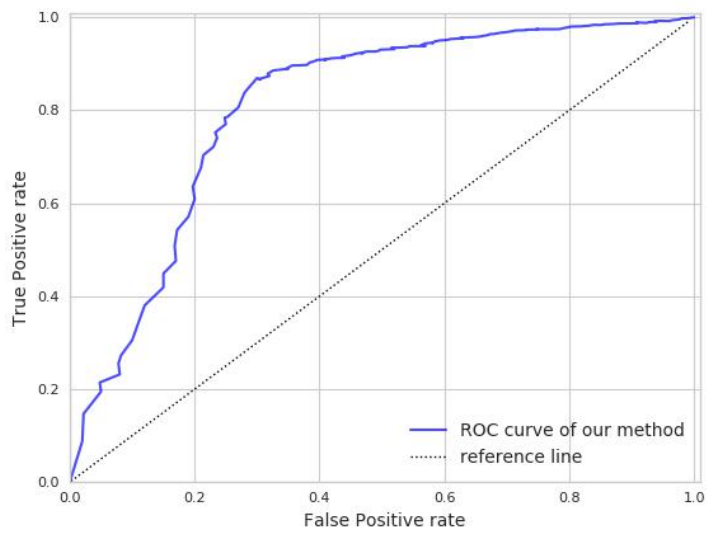

Fig. 5. ROC curve of our presented method for detecting cardiovascular vulnerable plaques.

Also, we evaluate our six-scale anchors approach to prove it has better performance than the conventional one-scale and three-scale approaches. The results are shown in table 3. A sample detection result of our proposed six-scale Faster R-CNN is shown in figure 6 (right).
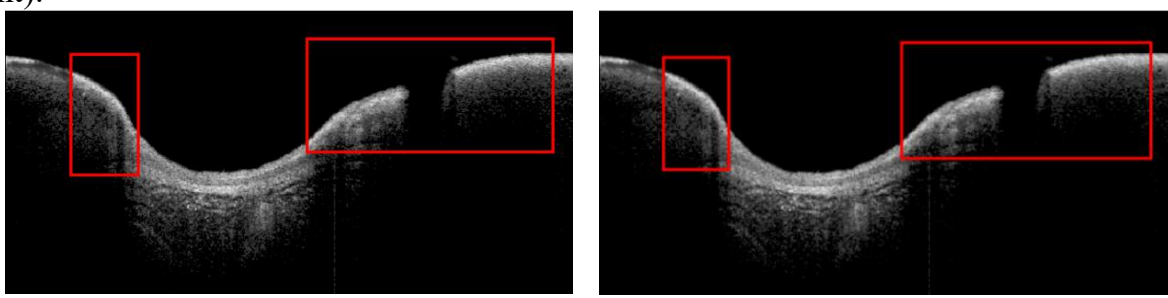

Fig. 6. Ground Truth image (left) and Faster R-CNN predicted result (right).

Table 3. AP of Faster R-CNN when using different scales and ratios.

\begin{tabular}{|c|c|c|c|}
\hline Settings & Anchor scales & Aspect ratios & $\mathrm{AP}(\%)$ \\
\hline 1 scale, 1 ratio & $128^{2}$ & $1: 1$ & 79.6 \\
\hline 1 scale, 1 ratio & 256 & $1: 1$ & 78.3 \\
\hline 1 scales, 3 ratios & $128^{2}$ & $\{1: 1,1: 2,2: 1\}$ & 81.2 \\
\hline 1 scale, 3 ratios & $256^{2}$ & $\{1: 1,1: 2,2: 1\}$ & 80.2 \\
\hline 3 scales, 1 ratio & $\left\{128^{2}, 256^{2}, 512^{2}\right\}$ & $1: 1$ & 81.7 \\
\hline 3 scales, 3 ratios & $\left\{128^{2}, 256^{2}, 512^{2}\right\}$ & $\{1: 1,1: 2,2: 1\}$ & 82.3 \\
\hline $\begin{array}{c}6 \text { scales, } 3 \text { ratios(our } \\
\text { method) }\end{array}$ & $\begin{array}{c}\left\{12^{2}, 16^{2}, 32^{2}, 64^{2}, 128^{2}, 25\right. \\
\left.6^{2}\right\}\end{array}$ & $\{1: 1,1: 2,2: 1\}$ & 83.4 \\
\hline
\end{tabular}




\section{Conclusion}

In this paper, we propose a novel cardiovascular vulnerable plaque detection method based on deep convolution neural networks. In the first stage we experiment on different thresholds to attain the best segmentation result, in the second stage we design an improved Faster R-CNN which has six-scale anchors to do precise detection. Experimental results on the CCCV2017 Cardiovascular Vulnerable Plaque Detection Challenge demonstrate the proposed method has superior performance than the $1^{\text {st }}$ team of the challenge. The Recall rate, Precision rate, IoU rate and Total score of our method are $0.94,0.885,0.913$ and 0.913 respectively, AP of the designed Faster R-CNN is $83.4 \%$, higher than conventional onescale and three-scale approaches. We believe our proposed method would be a very powerful tool for clinical cardiovascular vulnerable plaques diagnosis.

\section{Acknowledgments}

Research is supported by the National Natural Science Foundation of China under Grant U1613214 and in part supported by the Fundamental Research Funds for the Central Universities of China under Grant N170402008, by the Fund for the Shenyang City Science and Technology Plan under Grant F16-2051-04, by the 111 Project under Grant B16009. We thank all the CCCV2017 Cardiovascular OCT Vulnerable Plaque Detection Challenge organizers with special acknowledgments to Dr. Jianan Li, who comes from Xian Institute of Optics and Fine Mechanics, Chinese Academy of Sciences.

\section{References}

1. Fleg L, Stone W and Fayad A 2012 Detection of high-risk atherosclerotic plaque: report of the NHLBI Working Group on current status and future directions. JACC Cardiovasc Imaging 5(9) 941-955.

2. Ambrose A and Srikanth S 2010 Vulnerable plaques and patients:improving prediction of future coronary events. Am J Med 123(1) 10-16.

3. Takashi K, Takashi A and Junya S 2013 OCT compared with IVUS in a coronary lesion assessment: The OPUSCLASS Study. JACC Cardiovascular Imaging 6(10) 1095-1104.

4. Wang S, Luo J and Ling H 2013 Computer-aided diagnosis data platform by using medical imaging. Chin. J. Biomed. Eng 32(1) 105.

5. Valgimigli M, Agostoni P and Serruvs PW 2007 Acute coronary syndromes: an emphasis shift from treatment to prevention; and the enduring challenge of vulnerable plaque detection in the cardiac catheterization laboratory. Cardiovasic Med (Hagerstown) 8(4) 221-229.

6. Chen X 2014 Research on the Application of Sparse Representation Theory in Medical Image Processing and Analysis (Wuhan: Huazhong University of Science and Technology Press) p 4-10.

7. Keras Documentation/Image Preprocessing. https://keras.io/preprocessing/image/. last accessed 2018/08/14.

8. Ronneberger O, Fischer $\mathrm{P}$ and Brox T 2015 Int. Conf. on Medical image Computing and Computer-Assisted Intervention (Munich) pp.234-241. 
9. Li X, Chen H, Qi X, Dou Q, Fu C and Heng P 2018 H-DenseUNet: hybrid densely connected UNet for liver and tumor segmentation from CT volumes. IEEE Trans Med Imaging PP(99): 1-1.

10. Dong H, Yang G, Liu F, Mo Y and Guo Y 2017 Automatic brain tumor detection and segmentation using U-Net based fully convolutional networks. Conf. on Medical Image Understanding and Analysis.

11. Girshick R 2015 Fast R-CNN. IEEE Int. Conf. on Computer Vision.

12. Ren S, He K, Girshick R and Sun J 2015 Faster R-CNN: towards real-time object detection with region proposal networks. Conf. on Neural Information Processing Systems (Montreal) p 91-99.

13. He K, Zhang X, Ren S and Sun J 2015 Deep residual learning for image recognition. arXiv. 1512.03385 . 